\title{
Inflammatory links between obesity and metabolic disease
}

\author{
Carey N. Lumeng ${ }^{1,2}$ and Alan R. Saltiel2,3,4
}

1Department of Pediatrics and Communicable Diseases, ${ }^{2}$ Department of Molecular and Integrative Physiology, and ${ }^{3}$ Department of Internal Medicine, University of Michigan Medical School, Ann Arbor, Michigan, USA. ${ }^{4}$ Life Sciences Institute, University of Michigan, Ann Arbor, Michigan, USA.

\begin{abstract}
The obesity epidemic has forced us to evaluate the role of inflammation in the health complications of obesity. This has led to a convergence of the fields of immunology and nutrient physiology and the understanding that they are inextricably linked. The reframing of obesity as an inflammatory condition has had a wide impact on our conceptualization of obesity-associated diseases. In this Review, we highlight the cellular and molecular mechanisms at play in the generation of obesity-induced inflammation. We also emphasize how defining the immune regulation in metabolic tissues has broadened the understanding of the diversity of inflammatory responses.
\end{abstract}

\section{Introduction}

The burden of obesity on health extends across multiple organ systems and diseases. While its impact on tissues involved in nutrient regulation is manifest in the development of insulin resistance and type 2 diabetes, there are also unexpected connections between obesity and the risk of cancer and pulmonary diseases. Over the past decade, the search for a potential unifying mechanism behind the pathogenesis of obesity-associated diseases has revealed a close relationship between nutrient excess and derangements in the cellular and molecular mediators of immunity and inflammation. This has given birth to the concept of "metainflammation" (1) to describe the chronic low-grade inflammatory response to obesity. We present here a broad overview of the links between obesity and immune responses with a focus on metabolic disease and argue that the intersection between the pathways that control nutrient metabolism and inflammatory responses may be broadly applicable to our understanding of inflammation and the immune system.

\section{The nature of obesity-induced inflammation}

Inflammation is a coordinated response to harmful stimuli, with the goal of returning the system back to a normal baseline. The inflammatory response triggered by obesity involves many components of the classical inflammatory response to pathogens and includes systemic increases in circulating inflammatory cytokines and acute phase proteins (e.g., C-reactive protein), recruitment of leukocytes to inflamed tissues, activation of tissue leukocytes, and generation of reparative tissue responses (e.g., fibrosis; ref. 2). However, the nature of obesity-induced metainflammation is unique compared with other inflammatory paradigms (e.g., infection, autoimmune disease) in several key aspects. The chronic nature of obesity produces a tonic low-grade activation of the innate immune system that affects steady-state measures of metabolic homeostasis over time. Childhood obesity may place individuals at risk for lifelong metainflammation, since inflammatory markers are elevated in obese children as young as 3 years old (3). Superimposed on this chronic inflammation are recurrent acute episodes of nutrition-related immune activation induced by nutrient availability (fasting or high-fat meals) (4-6).

Conflict of interest: The authors have declared that no conflict of interest exists. Citation for this article: J Clin Invest. 2011;121(6):2111-2117. doi:10.1172/JCI57132.
In addition, the multi-organ involvement of obesity-induced inflammation is unique and presents a challenge to researchers attempting to tease out disease mechanisms in complex metabolic systems (ref. 7 and Figure 1). It is clear that inflammation participates in the link between obesity and disease. Non-biased assessments of gene expression networks in adipose tissue identify a robust pattern of overexpressed inflammatory genes associated with obesity and metabolic disease and enriched for macrophage genes $(8,9)$. Multiple inflammatory inputs contribute to metabolic dysfunction, including increases in circulating cytokines (10), decreases in protective factors (e.g., adiponectin; ref. 11), and communication between inflammatory and metabolic cells. For example, direct and paracrine signals from M1 classically activated macrophages can impair insulin signaling and adipogenesis in adipocytes, while unstimulated or M2 alternatively activated macrophages fail to generate these effects (12). Similar effects on adipocyte inflammation and glucose transport are generated by signals from activated conventional T cells such as IFN- $\gamma(13)$. In parallel, dysregulated macrophage-myocyte and macrophagehepatocyte signaling can influence insulin sensitivity $(14,15)$. While transient inflammatory states such as sepsis can have multiorgan effects, few other chronic inflammatory diseases are characterized by the features of pancreatic, liver, adipose, heart, brain, and muscle inflammation as is seen in obesity.

Immunity and the maintenance of metabolic homeostasis. In some cases, adaptive immune responses may be beneficial and help preserve metabolic homeostasis. All metabolic tissues contain resident populations of leukocytes present even in lean healthy animals, indicating that the immune system is poised to respond to nutrient-derived signals $(16,17)$. For example, the extent of adipose tissue macrophage (ATM) infiltration is dynamically altered with lipid flux in adipocytes in lean and obese states, and may serve to suppress lipolytic signals (4). ATMs are recruited to adipose tissue when chemokine or lipid release (lipolysis) is triggered and may function to promote lipid storage by suppressing lipolysis. These events could be classified as an inflammatory response, as it involves the acute recruitment of leukocytes to fat, but it lacks many of the cardinal signs of classic inflammation (dolor, rubor, calor, and tumor).

Reconciling these observations requires a more expansive view of what immunologic activation means beyond the classical proinflammatory paradigm. The diversity of ATM function sup- 
Body weight
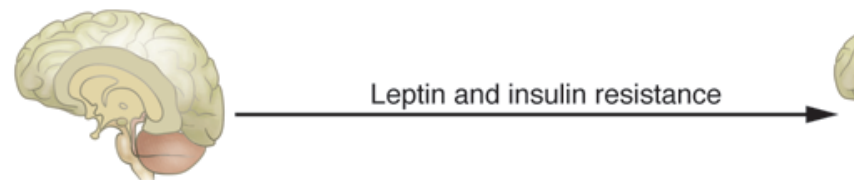

Brain

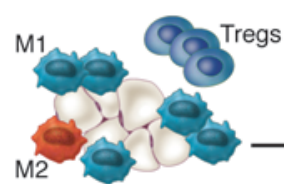

Adipose tissue

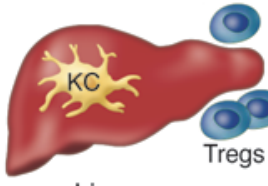

Liver

\section{,}

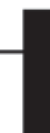
Insulin resistance

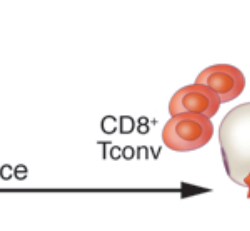
Hypothalmic
inflammation

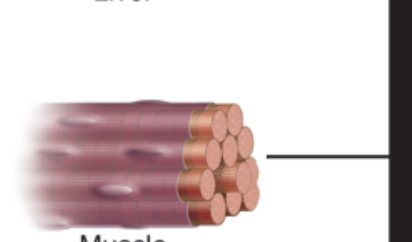

Muscle

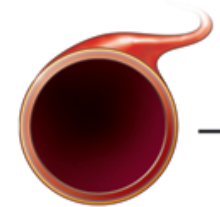

Blood vessels

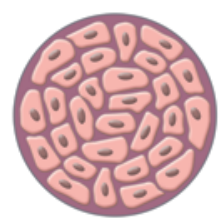

Pancreatic islets

\section{Figure 1}

Cellular mediators of inflammation and immunity in obesity. The multisystem effects of obesity are linked to an imbalance in homeostatic and proinflammatory immune responses. Obesity triggers inflammatory pathways in the brain and adipose tissue that dysregulate physiological responses that maintain insulin and leptin sensitivity. Over time, ectopic lipid accumulation in muscle, liver, and blood vessels activates tissue leukocytes, contributes to organ-specific disease, and exacerbates systemic insulin resistance. Cellular- and cytokine-mediated inflammation in pancreatic islets accelerates the progression toward diabetes. FFA, free FA; INS, insulin; KC, Kupffer cell; Tconv, conventional CD4+ T cells. macrophage activation state (M2) that promotes fibrotic responses and attenuation of classical NF-кB-dependent activation pathways. While ATMs likely assume a number of states along the M1/M2 spectrum depending on fat depot location and nutritional status $(19,20)$, increasing adiposity results in a shift in the inflammatory profile of ATMs as a whole from an M2 state to one in which classical M1 proinflammatory signals predominate (21-23).

Unique molecular links between the $\mathrm{M} 1 / \mathrm{M} 2$ polarization axis and metabolism suggest that the homeostatic regulation of nutrient utilization and the immune system are coupled together. The M2 activation state is intrinsically linked to the activity of PPAR $\delta$ and PPAR $\gamma$, well-known regulators of lipid metabolism and mitochondrial activity (24). Ppard- and Ppargknockout mice fail to generate an M2 activation state and are more susceptible to the M1-skewed inflammation that accompanies diet-induced obesity (DIO) in the liver and adipose tissue $(17,25,26)$. Physiologic enhancement of the M2 pathways (e.g., eosinophil recruitment in parasitic infection) also appears to be capable of reducing metainflammation and improving insulin sensitivity (27).

In fat, the M2 state of resident ATMs is maintained by cytokine production (e.g., IL-4) from unique natural helper lymphocytes and eosinophils; the recruitment of these cells to fat is suppressed in the obese environment $(27,28)$. This demonstrates that maintaining metabolic homeostasis requires a balanced immune response and an integrated network of multiple cell types. Adipose tissue also contains potent tolerogenic $\mathrm{CD}^{+}$Tregs that are downregulated by obesity, a potential initiating event in metainflammation $(13,29)$. Likewise, there appear to be innate systems by which nutrient signals are utilized to self-limit inflammation. For example, the obesity-induced increase in expression of GPR120, an omega-3 fatty acid (FA) receptor on macrophages capable of attenuating M1 macrophage activation and increasing M2 gene expression, limits inflammation; it is possible that this mechanism might be exploited in future drug development (30). ports this broad view, as does the observation that leukocytes adopt a wide range of activation states dependent upon the local stimuli (18). Upon stimulation by LPS and IFN- $\gamma$, macrophages assume a classical proinflammatory activation state (M1) that generates bactericidal or Th1 responses typically associated with obesity. In contrast, Th 2 cytokines such as IL-4 and IL-13 generate an alternative

\section{Tissue specific proinflammatory changes with obesity}

The discovery of ATM activation with obesity sparked a wave of interest into how immune responses intersect with obesity $(31,32)$. We know now that the dynamic regulation of inflammatory cells with obesity is not limited to fat and that inflammatory and metabolic signals converge in a myriad of contexts. This provides new 
opportunities to understand the pathogenesis of many organ-specific diseases associated with obesity (Figure 1).

Pancreatic islets. Relevant to type 2 diabetes is the demonstration that inflammation in pancreatic islets can reduce insulin secretion and trigger $\beta$ cell apoptosis leading to decreased islet mass, critical events in the progression to diabetes $(33,34)$. The mediators of these effects are multifactorial and likely involve cytokines produced by $\beta$ cells themselves (35). As in adipose tissue, macrophages accumulate in islets with DIO and may be a significant source of proinflammatory cytokines that block $\beta$ cell function (36). This point is often ignored in studies that manipulate macrophages by Cre-mediated recombination or BM transplantation and may be an underappreciated mechanism for protection from diabetes in many animal models.

Adipose tissue. Adipose tissue insulin resistance and dysfunctional lipid storage in adipocytes are sentinel events in the progression toward metabolic dysregulation with obesity. Forced expansion of adipose tissue by transgenic overexpression of the adipokine adiponectin prevents metabolic disease despite massive obesity (37). Impaired lipogenic/adipogenic capacity is associated with increased visceral fat in obese adolescents (38), and smaller omental adipocytes is a feature of metabolically healthy obese adults (39). These findings support the model that lipid "spillover" from fat promotes metabolic disease by fostering ectopic lipid deposition. Since an estimated excess of 20-30 million macrophages accumulate with each kilogram of excess fat in humans, one could argue that increased adipose tissue mass is de facto a state of increased inflammatory mass (40).

Inputs into this inflammatory response include ER stress, adipose tissue hypoxia, and adipocyte death (41-43). Since changes in ATM number and gene expression profile occur coincident with the development of insulin resistance $(41,44)$, it is possible that ATMs are merely effectors of a coordinated inflammatory response that includes the accumulation of $\mathrm{CD}^{+} \mathrm{T}$ cells, Th1polarized $\mathrm{CD}^{+} \mathrm{T}$ cells, and the loss of Tregs $(29,44)$. NK cells, NKT cells, and mast cells are also implicated in metainflammation $(40,45,46)$. Overall, our challenge in understanding adipose tissue inflammation will be to identify the temporal and spatial interactions between leukocytes in fat in the context of inflammatory initiation as well as their resolution.

Inflammation in liver and muscle. Nonalcoholic fatty liver disease (NAFLD) is a strong risk factor for insulin resistance, nonalcoholic steatohepatitis, and dyslipidemia, independent of visceral adiposity (47). Many of the signaling pathways involved in both inflammation and metabolism are elevated in steatotic liver (e.g., JNK, TLR4, ER stress). Similar to the effects of obesity on adipose tissue, NAFLD is associated with an increase in M1/Th1 cytokines and quantitative increases in immune cells (48-50). In addition, modulation of PPAR $\delta$-dependent M2 polarization pathways protects mice from NAFLD $(17,26)$. These effects may be mediated via Kupffer cells resident in the liver, or by unique cell populations recruited to the liver with obesity $(51)$.

There is also evidence of increased inflammatory cytokine production and increased inflammation in skeletal muscle in obesity (52). Myocytes have the capacity to respond to inflammatory signals via pattern recognition receptors (PRRs) such as TLR4 with direct metabolic effects (53). Muscle inflammation may be linked to infiltrating macrophages that are induced in obese muscle and have properties of M1 activation $(23,54)$. This topic is complicated by the fact that leukocyte trafficking of monocytes and macrophages is intrinsically linked to muscle injury and repair (55), increasing the challenge of de-convoluting the acute and chronic inflammatory changes in muscle with DIO.

Hypothalamic inflammation and obesity. Human genome wide association studies have identified loci near or within numerous neuronal genes that affect BMI, suggesting that variation in the central control of metabolism plays a prominent role in genetic obesity risk (56). Lipid infusion and a high-fat diet (HFD) activate hypothalamic inflammatory signaling pathways, resulting in increased food intake and nutrient storage (57). With DIO, metabolites such as diacylglycerols and ceramides accumulate in the hypothalamus and induce leptin and insulin resistance in the CNS $(58,59)$. Part of this effect is mediated by saturated FAs, which activate neuronal JNK and NF-אB signaling pathways with direct effects on leptin and insulin signaling (60). Disruption of signaling through TLR4/MyD88, IKK $\beta / \mathrm{NF}-\kappa \mathrm{B}$, and ER stress pathways in neurons protects mice from DIO and its downstream metabolic effects (60-62).

The effects of brain inflammation on the metabolic function of peripheral tissues are broad. Independent of obesity, hypothalamic inflammation can impair insulin release from $\beta$ cells, impair peripheral insulin action, and potentiate hypertension (63-65). Many of these effects are generated by signals from the sympathetic nervous system, which is also capable of inducing inflammatory changes in adipose tissue in response to neuronal injury (66). A future challenge is to understand how inflammatory signals in the brain generate responses that in some cases generate negative energy balance (anorexia), while in other cases generates positive energy balance (weight gain) (67).

The dynamic interplay between hypothalamic inflammation and obesity suggest additional targets for antiinflammatory therapies in obesity. A key extension of these observations is the potential that antiinflammatory pathways may counteract these CNS inflammatory events and improve leptin sensitivity. Recent evidence suggests that IL- 6 and IL-10 are involved in the exerciseinduced suppression of hyperphagia and suppress IKK $\beta / \mathrm{NF}-\kappa \mathrm{B}$ and ER stress in the brain (68). The IKK $\beta / \mathrm{NF}-\kappa \mathrm{B}$ inhibitor sodium salicylate is also capable of preventing the accumulation of ceramides in the hypothalamus with lipid infusion (58).

Development. Another intriguing possible link between inflammation and the risk for obesity involves events in early embryonic development. Epidemiologic and animal models have demonstrated a strong association between the prenatal and perinatal environment and obesity-associated diseases (69). The risk for obesity and metabolic disorders follows a U-shaped distribution based on birth weight, with increased risk in low- and high-birth-weight infants $(70,71)$. Since pregnancy represents a physiologic inflammatory state involving the innate and acquired immune system, inflammatory mechanisms may contribute to the in utero programming of nutrient metabolism (72). Maternal obesity is associated with endotoxemia and ATM accumulation that may affect the developing fetus (73). Placental inflammation is a characteristic of maternal obesity, a risk factor for obesity in offspring, and involves inflammatory macrophage infiltration that can alter the maternal-fetal circulation (74). Inflammatory disturbances in the placenta may alter the nutrient set points established early in life and predispose to an accelerated pattern of catch-up growth that contributes to the risk for later obesity, especially in low-birth-weight infants $(75,76)$. The concept that inflammatory networks can influence the predilection toward obesity is supported by the find- 


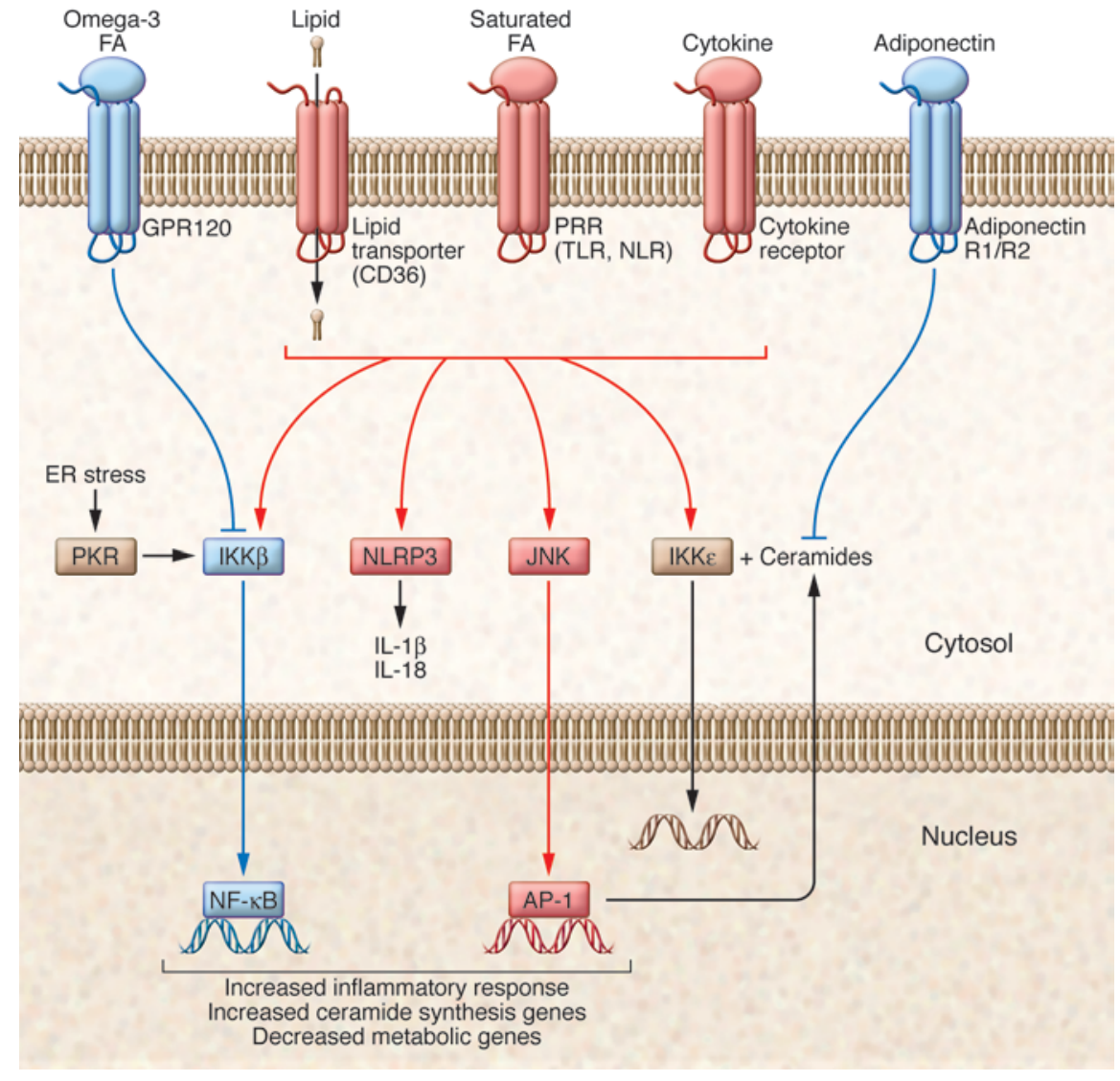

Figure 2

Molecular pathways at the interface between obesity and inflammation. Multiple signaling pathways participate in translating obesity-derived nutrient and inflammatory signals into a cellular response relevant in disease. These include proinflammatory (red) and antiinflammatory (blue) signals from the cell surface that integrate through many common intracellular pathways to generate the coordinated increase in inflammatory genes while repressing genes important in maintaining proper nutrient metabolism. PKR, RNA-dependent protein kinase.

ings that variation in obesity susceptibility between mouse strains is intrinsically linked to the inflammatory networks and leukocyte composition of adipose tissue established prior to HFD exposure (77). Overall, there is much to be learned about how maternal and paternal factors contribute to the epigenetic programming of metabolism genes that contribute to long-term effects on adult body weight $(78,79)$. It will be interesting to see whether inflammatory response genes are coordinately altered with metabolic gene networks based on the in utero environment.

\section{How does inflammation cause metabolic dysfunction?}

Many molecular signaling pathways have been described at the interface between inflammation and metabolism (e.g., insulin receptor signaling in macrophages; ref. 80). Here we will highlight several prominent pathways that are coordinately regulated during obesity that translate a metabolic challenge into an inflammatory response and contribute to obesity-associated disease (Figure 2).

PRRs as metabolic sensors. PRRs are components of the innate immune system well known for their ability to sense foreign molecules (pathogen-associated molecular patterns) and initiate a defense response. However, the ability of PRRs to sense endogenous ligands induced in the obese state is now understood to be a trigger in obesity-associated inflammation. Of these PRRs, TLR4 has received the most attention, as this receptor can be activated by free FAs to generate proinflammatory signals and activate NF-кB (81). TLR4-deficient mice are protected from the inflammatory activation induced by obesity and demonstrate protection from insulin resistance induced by lipid infusion (82). Part of this effect is mediated by leukocytes (83), but there is clear evidence that direct effects of TLR4 activation in non-hematopoietic cells contribute to the metabolic phenotype (53). Adipose tissue expresses nearly all TLR family members, and TLR2-knockout mice are protected from high-fat DIO and insulin resistance, suggesting a broad role for TLRs in obesity and its associated morbidities $(84,85)$. Besides FAs, TLRs also sense and regulate gut microbes in a way that contributes to metabolism, as $\mathrm{Tl} 5^{-/-}$mice demonstrate obesity and insulin resistance related to alterations in the gut microbiome (86).

The Nod-like receptor (NLR) family of PRRs also sense obesity-induced signals in multiple contexts. NLRs are activated by danger signals from stressed or dying cells and mobilize leukocytes toward these stimuli to constrain tissue damage $(87,88)$. In macrophages, NLR activation stimulates the cryptopyrin/NLRP3 inflammasome to induce IL-1 $\beta$ and IL-18 production via caspase- 1 . These pathways contribute to pancreatic $\beta$ cell death with chronic hyperglycemia and affect diabetes progression (89). Caspase- 1 and IL- $1 \beta$ are also induced in adipose tissue with DIO, and Nlrp3-and Casp1-deficient mice demonstrate resistance to DIO-induced inflammation (90). The mechanism of this protective effect may be driven by alterations in the M1 activation of ATMs, as Nlrp3-knockout mice show decreased M1 and increased $M 2$ gene expression without quantitative changes in ATMs. If PRRs can broadly act as dual sensors of pathogenic and endogenous signals relevant to obesity, the potential pathways contributing to metainflammation may be vast.

$I K K \beta$ and NF-кB. The intracellular signals downstream of TLR activation occur via multiple pathways, which may be dependent on, or independent of, the adaptor protein MyD88. While MyD88dependent signaling contributes to hypothalamic inflammation with obesity (61), its role in other metabolic tissues is unclear, as $M y d 88^{-/-}$mice are more susceptible to insulin resistance with DIO (91). Activation of IKB kinase- $\beta$ (IKK $\beta$ ) occurs downstream of MyD88 and plays critical roles in inflammation in the liver, myeloid cells, and the hypothalamus in the obese state $(92,93)$. This wide range of action likely explains the insulin-sensitizing effect of salicylate, an IKK $\beta$ inhibitor that is currently in clinical trials for type 2 diabetes therapy $(94,95)$.

The ultimate endpoint for TLR/IKK $\beta$ signals is NF-KB-dependent activation of inflammatory gene transcription (96). In vivo imaging of NF- $\mathrm{\kappa B}$ activation identified adipose tissue and ATMs 


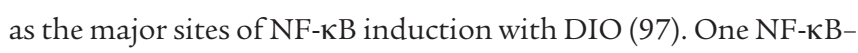
sensitive gene induced by HFD is the protein kinase Ikke, which appears to play a role in maintaining body weight and insulin resistance by suppressing thermogenesis (97). The temporal control of NF- $\kappa \mathrm{B}$ activation is also important, and there are unresolved issues differentiating the metabolic effects of acute and chronic NF- $\kappa \mathrm{B}$ activation. For example, acute exercise in lean adults stimulates muscle NF- $\mathrm{KB}$ activity correlating with the transient release of proinflammatory cytokines such as IL-6 (98).

Ceramides and intracellular lipids in inflammation and metabolism. The downstream effects of TLR4 activation are not limited to the activation of NF- $\mathrm{KB}$. A key common denominator between metabolism and inflammation may lie in the balance between intracellular lipid species such as ceramides and sphingolipids $(99,100)$. Inhibition of ceramide production blocks the ability of saturated FAs to induce insulin resistance (101). The induction of ceramide synthesis by LPS and saturated FA is dependent upon TLR4 in many metabolic tissues, including the hypothalamus and muscle, where ceramide production can inhibit insulin signaling through Akt (58). TLR4-mediated ceramide production in metabolic tissues is dependent on IKK $\beta$, as salicylates decrease ceramide levels in the liver, muscle, and hypothalamus.

The adipokine adiponectin has long been recognized to have positive benefits on multiple cell types to promote insulin sensitivity and deactivate proinflammatory pathways. Ceramide regulation may be a common denominator of these effects, as adiponectin stimulates ceramidase activity and modulates the balance between ceramides and sphingosine-1-phosphate (102). The influence of adiponectin on ceramide content in cells appears to be important for multiple tissues, as it guards against apoptosis of cardiomyocytes and $\beta$ cells (102). Adiponectin receptor-associated ceramidase activity may not be the only mechanism at play. Awazawa et al. found that insulin sensitivity was improved with adiponectin infusion in hepatocytes via IRS2 induction; however, this was not cell autonomous (103). Surprisingly, induction of IL-6 by adiponectin in macrophages generated this insulin-sensitizing effect; this was largely independent of adiponectin receptors R1 and R2.

$J N K$ and ER stress. In addition to NF- $\mathrm{KB}$, obesity also activates JNK in insulin-responsive tissues, probably through upstream pathways shared by IKK/NF- $\mathrm{BB}$ in response to stress signals such as FAs, insulin, hyperglycemia, and inflammatory cytokines (104). The differential contribution of JNK in hematopoietic and nonhematopoietic cells in obesity is well described compared with other inflammatory signaling components. While both JNK1 and JNK2 isoforms contribute to metabolic regulation, JNK1 has a more prominent role in the protection from DIO $(105,106)$. The effects of JNK1 in nonhematopoietic cells control body weight and energy expenditure. As with the IKK $\beta$ pathway, JNK1 also regulates hypothalamic signals; inactivation of JNK1 in the hypothalamus protects mice from DIO and recapitulates the reduced body weight phenotype seen in the whole body of JNK1-deficient animals (107). JNK1 inactivation in hematopoietic cells does not alter adiposity but is sufficient to attenuate obesity-induced inflammation with beneficial effects on metabolism $(108,109)$.

ER stress and the downstream activation of the molecular pathways governing the unfolded protein response appear to be closely tied to both JNK1 and IKK/NF-KB activation pathways in multiple metabolic tissues (e.g., hypothalamus and adipose tissue) (110). Widespread activation of ER stress signaling components and cascades (ATF6, PERK, IRE-1) is seen in obesity, and pharmacologic inhibition of ER stress can reverse metabolic dysfunction $(43,111)$. The double-stranded RNA-dependent protein kinase represents a PRR that sits at the interface between ER stress and nutrients to translate these signals into an inflammatory response through JNK (112). Further work is needed to establish how widespread ER stress is in other acute and chronic stress situations and how its mechanism overlaps with its role in the pathogenesis of atherosclerosis and foam cell biology (113).

\section{Antiinflammatory therapies for metabolic disease}

The observations above demonstrate potential new approaches toward the development of drugs that target inflammation to break the links between obesity and disease. Salsalate, a prodrug of salicylate, can attenuate IKK $\beta / \mathrm{NF}-\kappa \mathrm{B}$ activity and has been shown to improve glycemic control in patients with type 2 diabetes $(95,114)$. Preclinical studies have also demonstrated a metabolic benefit related to the antiinflammatory effects of targeting ER stress (111) and PPAR $\gamma$ (115). Blockade of inflammatory cytokine signals in metabolic disease also shows promise, as IL-1 receptor antagonists (anakinra) improve glycemia and $\beta$ cell function and decrease systemic inflammatory markers (116). In aggregate, TNF- $\alpha$ blockade with etanercept has not demonstrated significant improvements in insulin sensitivity, but beneficial effects of TNF- $\alpha$ blockade on fasting glucose and circulating inflammatory cytokines have been observed, suggesting application in other obesity-related morbidities $(117,118)$.

\section{Conclusion}

The unexpected overlap between inflammatory and metabolic sensors and their downstream tissue responses indicates that inflammation plays a crucial role in the many complications of obesity. However, there remain many contradictions that have yet to be sorted out, such as how hypothalamic inflammation causes both obesity and anorexia, the role of the innate immune system in maintaining obesity, and the teleological reasons for obesity-dependent inflammation, among others. We feel that answering these questions requires approaches that allow a higher-resolution view of the different cellular mediators (e.g., macrophages, adipocytes, and lymphocytes) in fat that contribute to disease phenotypes. A second challenge will be to establish the differences and similarities between acute inflammatory signals and chronic activation of these pathways in diseases such as obesity. This temporal question is also a critical one for immunologists, who are employing systems biology approaches to tease out the dynamic network effects of transient versus persistent stimuli (119). These efforts provide opportunities to continue to revise our understanding of the nature of metainflammation in the hope of modifying it to prevent and treat disease.

\section{Acknowledgments}

The Obesity Review Series is supported in part by unrestricted educational grants from Merck \& Co. and the Life Sciences Institute of the University of Michigan. This work was supported by NIH National Institute of Diabetes and Digestive and Kidney Diseases grants to C.N. Lumeng (R01DK090262, K08DK078851) and A.R. Saltiel (R01DK060591).

Address correspondence to: Carey Lumeng, University of Michigan Medical School, Department of Pediatrics and Communicable Diseases, 4520B MSRB I, 1150 W Medical Center Drive, Ann Arbor, Michigan 48109-5652, USA. Phone: 734.936.4200; Fax: 734.763.9512; E-mail: clumeng@umich.edu. 
1. Hotamisligil GS. Inflammation and metabolic disorders. Nature. 2006;444(7121):860-867.

2. Spencer $M$, et al. Adipose tissue macrophages in insulin-resistant subjects are associated with collagen VI and fibrosis and demonstrate alternative activation. Am J Physiol Endocrinol Metab. 2010;299(6):E1016-E1027.

3. Skinner AC, Steiner MJ, Henderson FW, Perrin EM. Multiple markers of inflammation and weight status: cross-sectional analyses throughout childhood. Pediatrics. 2010;125(4):e801-e809.

4. Kosteli A, et al. Weight loss and lipolysis promote a dynamic immune response in murine adipose tissue. J Clin Invest. 2010;120(10):3466-3479.

5. Alipour A, Elte JW, van Zaanen HC, Rietveld AP, Cabezas MC. Postprandial inflammation and endothelial dysfuction. Biochem Soc Trans. 2007; 35(pt 3):466-469

6. Blackburn $P$, et al. Postprandial variations of plasma inflammatory markers in abdominally obese men. Obesity (Silver Spring). 2006;14(10):1747-1754

7. Butler AA, Kozak LP. A recurring problem with the analysis of energy expenditure in genetic models expressing lean and obese phenotypes. Diabetes. 2010 59(2):323-329.

8. Chen Y, et al. Variations in DNA elucidate molecular networks that cause disease. Nature. 2008 452(7186):429-435.

9. Emilsson V, et al. Genetics of gene expression and its effect on disease. Nature. 2008;452(7186):423-428.

10. Hotamisligil GS, Arner P, Caro JF, Atkinson RL, Spiegelman BM. Increased adipose tissue expression of tumor necrosis factor-alpha in human obesity and insulin resistance. J Clin Invest. 1995; 95(5):2409-2415

11. Hotta $\mathrm{K}$, et al. Plasma concentrations of a novel, adipose-specific protein, adiponectin, in type 2 diabetic patients. Arterioscler. Thromb Vasc Biol. 2000 20(6):1595-1599.

12. Lumeng CN, Del Proposto JB, Westcott DJ, Saltiel AR. Phenotypic switching of adipose tissue macrophages with obesity is generated by spatiotemporal differences in macrophage subtypes. Diabetes. 2008;57(12):3239-3246.

13. Feuerer $M$, et al. Lean, but not obese, fat is enriched for a unique population of regulatory $\mathrm{T}$ cells that affect metabolic parameters. Nat Med. 2009;15(8):930-939.

14. Varma V, et al. Muscle inflammatory response and insulin resistance: synergistic interaction between macrophages and fatty acids leads to impaired insulin action. Am J Physiol Endocrinol Metab. 2009; 296(6):E1300-E1310.

15. Huang W, et al. Depletion of liver Kupffer cells prevents the development of diet-induced hepatic steatosis and insulin resistance. Diabetes. 2010;59(2):347-357.

16. Lumeng CN, Bodzin JL, Deyoung SM, Saltiel AR Increased inflammatory properties of adipose tissue macrophages recruited during diet induced obesity. Diabetes. 2007;6(1):16-23.

17. Odegaard JI, et al. Alternative M2 activation of Kupffer cells by PPARdelta ameliorates obe sity-induced insulin resistance. Cell Metab. 2008; 7(6):496-507.

18. Mantovani A, Sica A, Sozzani S, Allavena P, Vecchi A, Locati M. The chemokine system in diverse forms of macrophage activation and polarization. Trends Immunol. 2004;25(12):677-686.

19. Shaul ME, Bennett G, Strissel KJ, Greenberg AS, Obin MS. Dynamic, M2-like remodeling phenotypes of $\mathrm{CD} 11 \mathrm{c}+$ adipose tissue macrophages during high-fat diet-induced obesity in mice. Diabetes. 2010;59(5):1171-1181.

20. Zeyda M, Gollinger K, Kriehuber E, Kiefer FW, Neuhofer A, Stulnig TM. Newly identified adipose tissue macrophage populations in obesity with distinct chemokine and chemokine receptor expression.
Int J Obes (Lond). 2010;34(12):1684-1694.

21. Aron-Wisnewsky J, et al. Human adipose tissue macrophages: $\mathrm{m} 1$ and $\mathrm{m} 2$ cell surface markers in subcutaneous and omental depots and after weight loss. J Clin Endocrinol Metab. 2009;94(11):4619-4623.

22. Lumeng CN, Bodzin JL, Saltiel AR. Obesity induces a phenotypic switch in adipose tissue macrophage polarization. J Clin Invest. 2007;117(1):175-184.

23. Patsouris D, Li PP, Thapar D, Chapman J, Olefsky JM, Neels JG. Ablation of CD11c-positive cells normalizes insulin sensitivity in obese insulin resistant animals. Cell Metab. 2008;8(4):301-309.

24. Odegaard JI, Chawla A. Alternative macrophage activation and metabolism. Annu Rev Pathol. 2011; 6:275-297.

25. Odegaard JI, et al. Macrophage-specific PPARgamma controls alternative activation and improves insulin resistance. Nature. 2007;447(7148):1116-1120.

26. Kang K, et al. Adipocyte-derived Th2 cytokines and myeloid PPARdelta regulate macrophage polarization and insulin sensitivity. Cell Metab. 2008;7(6):485-495.

27. Wu D, et al. Eosinophils sustain adipose alternatively activated macrophages associated with glucose homeostasis. Science. 2011;332(6026):243-247.

28. Moro $\mathrm{K}$, et al. Innate production of $\mathrm{T}(\mathrm{H}) 2$ cytokines by adipose tissue-associated c-Kit(+)Sca-1(+) lymphoid cells. Nature. 2010;463(7280):540-544.

29. Winer S, et al. Normalization of obesity-associated insulin resistance through immunotherapy: CD4+ T cells control glucose homeostasis. Nat Med. 2009; 15(8):921-929.

30. Oh DY, et al. GPR120 is an omega-3 fatty acid receptor mediating potent anti-inflammatory and insulin-sensitizing effects. Cell. 2010;142(5):687-698.

31. $\mathrm{Xu} \mathrm{H}$, et al. Chronic inflammation in fat plays a crucial role in the development of obesity-related insulin resistance. J Clin Invest. 2003;112(12):1821-1830.

32. Weisberg SP, McCann D, Desai M, Rosenbaum M, Leibel RL, Ferrante AW Jr. Obesity is associated with macrophage accumulation in adipose tissue. J Clin Invest. 2003;112(12):1796-1808.

33. Ehses JA, Boni-Schnetzler M, Faulenbach M, Donath MY. Macrophages, cytokines and beta-cell death in Type 2 diabetes. Biochem Soc Trans. 2008; 36(pt 3):340-342

34. Donath MY, Boni-Schnetzler M, Ellingsgaard $\mathrm{H}$, Halban PA, Ehses JA. Cytokine production by islets in health and diabetes: cellular origin, regulation and function. Trends Endocrinol Metab. 2010;21(5):261-267.

35. Maedler K, et al. Glucose-induced beta cell production of IL-1beta contributes to glucotoxicity in human pancreatic islets. J Clin Invest. 2002; 110(6):851-860.

36. Ehses JA, et al. Increased number of islet-associated macrophages in type 2 diabetes. Diabetes. 2007;56(9):2356-2370

37. Kim JY, et al. Obesity-associated improvements in metabolic profile through expansion of adipose tissue. J Clin Invest. 2007;117(9):2621-2637.

38. Kursawe R, et al. Cellularity and adipogenic profile of the abdominal subcutaneous adipose tissue from obese adolescents: association with insulin resistance and hepatic steatosis. Diabetes. 2010;59(9):2288-2296

39. O'Connell J, et al. The relationship of omental and subcutaneous adipocyte size to metabolic disease in severe obesity. PLoS One. 2010;5(4):e9997.

40. O'Rourke RW, et al. Depot-specific differences in inflammatory mediators and a role for NK cells and IFN-gamma in inflammation in human adipose tissue. Int J Obes (Lond). 2009;33(9):978-990.

41. Strissel KJ, et al. Adipocyte death, adipose tissue remodeling, and obesity complications. Diabetes. 2007;56(12):2910-2918

42. Hosogai N, et al. Adipose tissue hypoxia in obesity and its impact on adipocytokine dysregulation.
Diabetes. 2007;56(4):901-911.

43. Ozcan U, et al. Endoplasmic reticulum stress links obesity, insulin action, and type 2 diabetes. Science. 2004;306(5695):457-461.

44. Nishimura S, et al. CD8+ effector T cells contribute to macrophage recruitment and adipose tissue inflammation in obesity. Nat Med.2009:15(8):914-920.

45. Liu J, et al. Genetic deficiency and pharmacological stabilization of mast cells reduce diet-induced obesity and diabetes in mice. Nat Med. 2009;15(8):940-945.

46. Ohmura K, et al. Natural killer T cells are involved in adipose tissues inflammation and glucose intolerance in diet-induced obese mice. Atertioscler Thromb Vasc Biol. 2010;30(2):193-199.

47. Fabbrini $\mathrm{E}$, et al. Intrahepatic fat, not visceral fat, is linked with metabolic complications of obesity. Proc Natl Acad Sci U S A. 2009;106(36):15430-15435.

48. Li Z, Soloski MJ, Diehl AM. Dietary factors alter hepatic innate immune system in mice with nonalcoholic fatty liver disease. Hepatology. 2005; 42(4):880-885.

49. Kremer M, Hines IN, Milton RJ, Wheeler MD. Favored $\mathrm{T}$ helper 1 response in a mouse model of hepatosteatosis is associated with enhanced T cellmediated hepatitis. Hepatology. 2006;44(1):216-227.

50. Baffy G. Kupffer cells in non-alcoholic fatty liver disease: the emerging view. J Hepatol. 2009; 51(1):212-223.

51. Obstfeld AE, et al. C-C chemokine receptor 2 (CCR2) regulates the hepatic recruitment of myeloid cells that promote obesity-induced hepatic steatosis. Diabetes. 2010;59(4):916-925.

52. Saghizadeh M, Ong JM, Garvey WT, Henry RR, Kern PA. The expression of TNF alpha by human muscle. Relationship to insulin resistance. J Clin Invest. 1996;97(4):1111-1116.

53. Frisard MI, et al. Toll-like receptor 4 modulates skeletal muscle substrate metabolism. Am J Physiol Endocrinol Metab. 2010;298(5):E988-E998.

54. Hong EG, et al. Interleukin-10 prevents dietinduced insulin resistance by attenuating macrophage and cytokine response in skeletal muscle. Diabetes. 2009;58(11):2525-2535

55. Arnold L, et al. Inflammatory monocytes recruited after skeletal muscle injury switch into antiinflammatory macrophages to support myogenesis. J Exp Med. 2007;204(5):1057-1069.

56. Speliotes EK, et al. Association analyses of 249,796 individuals reveal 18 new loci associated with body mass index. Nat Genet. 2010;42(11):937-948.

57. Thaler JP, Schwartz MW. Minireview: Inflammation and obesity pathogenesis: the hypothalamus heats up. Endocrinology. 2010;151(9):4109-4115.

58. Holland WL, et al. Lipid-induced insulin resistance mediated by the proinflammatory receptor TLR4 requires saturated fatty acid-induced ceramide biosynthesis in mice. JClin Invest. 2011;121(5):1858-1870.

59. Benoit SC, et al. Palmitic acid mediates hypothalamic insulin resistance by altering PKC-theta subcellular localization in rodents. J Clin Invest. 2009; 119(9):2577-2589.

60. Zhang X, Zhang G, Zhang H, Karin M, Bai H, Cai D. Hypothalamic IKKbeta/NF-kappaB and ER stress link overnutrition to energy imbalance and obesity. Cell. 2008;135(1):61-73.

61. Kleinridders A, et al. MyD88 signaling in the CNS is required for development of fatty acid-induced leptin resistance and diet-induced obesity. Cell Metab. 2009;10(4):249-259.

62. Ozcan L, et al. Endoplasmic reticulum stress plays a central role in development of leptin resistance. Cell Metab. 2009;9(1):35-51.

63. Kang YM, et al. Brain nuclear factor-kappa B activation contributes to neurohumoral excitation in angiotensin II-induced hypertension. Cardiovasc Res. 2009;82(3):503-512.

64. Calegari VC, et al. Inflammation of the hypothalamus leads to defective pancreatic islet function. 
J Biol Chem. 2011;286(15):12870-12880.

65. Purkayastha S, Zhang H, Zhang G, Ahmed Z, Wang Y, Cai D. Neural dysregulation of peripheral insulin action and blood pressure by brain endoplasmic reticulum stress. Proc Natl Acad Sci U S A. 2011;108(7):2939-2944.

66. Wang YY, Lin SY, Chuang YH, Chen CJ, Tung KC, Sheu WH. Adipose proinflammatory cytokine expression through sympathetic system is associated with hyperglycemia and insulin resistance in a rat ischemic stroke model. Am J Physiol Endocrinol Metab. 2011;300(1):E155-E163.

67. Thaler JP, Choi SJ, Schwartz MW, Wisse BE. Hypothalamic inflammation and energy homeostasis: resolving the paradox. Front Neuroendocrinol. 2010;31(1):79-84.

68. Ropelle ER, et al. IL-6 and IL-10 anti-inflammatory activity links exercise to hypothalamic insulin and leptin sensitivity through IKKbeta and ER stress inhibition. PLoS Biol. 2010;8(8):e1000465.

69. Monasta L, et al. Early-life determinants of overweight and obesity: a review of systematic reviews. Obes Rev. 2010;11(10):695-708.

70. Forsen T, Eriksson J, Tuomilehto J, Reunanen A, Osmond C, Barker D. The fetal and childhood growth of persons who develop type 2 diabetes. Ann Intern Med. 2000;133(3):176-182.

71. Baker JL, Olsen LW, Sorensen TI. Weight at birth and all-cause mortality in adulthood. Epidemiology. 2008;19(2):197-203.

72. Challis JR, Lockwood CJ, Myatt L, Norman JE, Strauss JF 3rd, Petraglia F. Inflammation and pregnancy. Reprod Sci. 2009;16(2):206-215.

73. Basu S, et al. Pregravid obesity associates with increased maternal endotoxemia and metabolic inflammation. Obesity (Silver Spring). 2011; 19(3):476-482.

74. Frias AE, et al. Maternal high-fat diet disturbs uteroplacental hemodynamics and increases the frequency of stillbirth in a nonhuman primate model of excess nutrition [published online ahead of print March 29, 2011]. Endocrinology. doi:10.1210/en.2010-1332.

75. Jimenez-Chillaron JC, Patti ME. To catch up or not to catch up: is this the question? Lessons from animal models. Curr Opin Endocrinol Diabetes Obes. 2007;14(1):23-29.

76. Isganaitis E, et al. Accelerated postnatal growth increases lipogenic gene expression and adipocyte size in low-birth weight mice. Diabetes. 2009; 58(5):1192-1200.

77. Mori MA, et al. A systems biology approach identifies inflammatory abnormalities between mouse strains prior to development of metabolic disease. Diabetes. 2010;59(11):2960-2971.

78. Carone BR, et al. Paternally induced transgenerational environmental reprogramming of metabolic gene expression in mammals. Cell. 2010; 143(7):1084-1096.

79. Ng SF, Lin RC, Laybutt DR, Barres R, Owens JA, Morris MJ. Chronic high-fat diet in fathers programs beta-cell dysfunction in female rat offspring. Nature. 2010;467(7318):963-966.

80. Baumgartl J, et al. Myeloid lineage cell-restricted insulin resistance protects apolipoproteinE-deficient mice against atherosclerosis. Cell Metab. 2006 . 3(4):247-256.

81. Fessler MB, Rudel LL, Brown JM. Toll-like receptor signaling links dietary fatty acids to the metabolic syndrome. Curr Opin Lipidol. 2009;20(5):379-385.

82. Shi H, Kokoeva MV, Inouye K, Tzameli I, Yin H, Flier JS. TLR4 links innate immunity and fatty acid-induced insulin resistance. J Clin Invest. 2006; 116(11):3015-3025

83. Saberi M, et al. Hematopoietic cell-specific deletion of toll-like receptor 4 ameliorates hepatic and adipose tissue insulin resistance in high-fat-fed mice. Cell Metab. 2009;10(5):419-429.

84. Poulain-Godefroy O, et al. Inflammatory role of Toll-like receptors in human and murine adipose tissue. Mediators Inflamm. 2010;2010:823486.

85. Himes RW, Smith CW. Tlr2 is critical for dietinduced metabolic syndrome in a murine model. FASEB J. 2010;24(3):731-739.

86. Vijay-Kumar M, et al. Metabolic syndrome and altered gut microbiota in mice lacking Toll-like receptor 5. Science. 2010;328(5975):228-231.

87. Chen G, Shaw MH, Kim YG, Nunez G. NOD-like receptors: role in innate immunity and inflammatory disease. Annu Rev Pathol. 2009;4:365-398.

88. Schroder K, Zhou R, Tschopp J. The NLRP3 inflammasome: a sensor for metabolic danger? Science. 2010;327(5963):296-300.

89. Zhou R, Tardivel A, Thorens B, Choi I, Tschopp J. Thioredoxin-interacting protein links oxidative stress to inflammasome activation. Nat Immunol. 2010;11(2):136-140.

90. Vandanmagsar B, et al. The NLRP3 inflammasome instigates obesity-induced inflammation and insulin resistance. Nat Med. 2011;17(2):179-188.

91. Hosoi T, Yokoyama S, Matsuo S, Akira S, Ozawa K. Myeloid differentiation factor 88 (MyD88)-deficiency increases risk of diabetes in mice. PLoS One. 2010;5(9):e12537.

92. Arkan MC, et al. IKK-beta links inflammation to obesity-induced insulin resistance. Nat Med. 2005;11(2):191-198.

93. Cai $\mathrm{D}$, et al. Local and systemic insulin resistance resulting from hepatic activation of IKK-beta and NF-kappaB. Nat Med. 2005;11(2):183-190.

94. Yuan M, et al. Reversal of obesity- and diet-induced insulin resistance with salicylates or targeted disruption of Ikkbeta. Science. 2001;293(5535):1673-1677.

95. Goldfine AB, Fonseca V, Jablonski KA, Pyle L, Staten MA, Shoelson SE. The effects of salsalate on glycemic control in patients with type 2 diabetes: a randomized trial. Ann Intern Med. 2010; 152(6):346-357.

96. Baker RG, Hayden MS, Ghosh S. NF-kappaB, inflammation, and metabolic disease. Cell Metab. 2011;13(1):11-22.

97. Chiang S-H, et al. The protein kinase IKKepsilon regulates energy balance in obese mice. Cell. 2009; 138(5):961-975.

98. Tantiwong $\mathrm{P}$, et al. NF-kappaB activity in muscle from obese and type 2 diabetic subjects under basal and exercise-stimulated conditions. Am J Physiol Endocrinol Metab. 2010;299(5):E794-E801.

99. Hannun YA, Obeid LM. Principles of bioactive lipid signalling: lessons from sphingolipids. Nat Rev Mol Cell Biol. 2008;9(2):139-150.

100.Summers SA. Sphingolipids and insulin resistance: the five Ws. Curr Opin Lipidol. 2010;21(2):128-135.

101. Holland WL, et al. Inhibition of ceramide synthesis ameliorates glucocorticoid-, saturated-fat-, and obesity-induced insulin resistance. Cell Metab.
2007;5(3):167-179.

102.Holland WL, et al. Receptor-mediated activation of ceramidase activity initiates the pleiotropic actions of adiponectin. Nat Med. 2011;17(1):55-63.

103. Awazawa $M$, et al. Adiponectin enhances insulin sensitivity by increasing hepatic IRS-2 expression via a macrophage-derived IL-6-dependent pathway. Cell Metab. 2011;13(4):401-412.

104.Solinas G, Karin M. JNK1 and IKKbeta: molecular links between obesity and metabolic dysfunction. FASEB J. 2010;24(8):2596-2611.

105. Hirosumi J, et al. A central role for JNK in obesity and insulin resistance. Nature. 2002; 420(6913):333-336.

106. Tuncman G, Hirosumi J, Solinas G, Chang L, Karin M, Hotamisligil GS. Functional in vivo interactions between JNK1 and JNK2 isoforms in obesity and insulin resistance. Proc Natl Acad Sci U S A. 2006;103(28):10741-10746.

107. Belgardt BF, et al. Hypothalamic and pituitary c-Jun $\mathrm{N}$-terminal kinase 1 signaling coordinately regulates glucose metabolism. Proc Natl Acad Sci U S A. 2010;107(13):6028-6033.

108.Solinas G, et al. JNK1 in hematopoietically derived cells contributes to diet-induced inflammation and insulin resistance without affecting obesity. Cell Metab. 2007;6(5):386-397.

109. Vallerie SN, Furuhashi M, Fucho R, Hotamisligil GS. A predominant role for parenchymal c-Jun amino terminal kinase (JNK) in the regulation of systemic insulin sensitivity. PLoS One. 2008;3(9):e3151.

110.Hotamisligil GS. Endoplasmic reticulum stress and the inflammatory basis of metabolic disease. Cell. 2010;140(6):900-917.

111.Ozcan U, et al. Chemical chaperones reduce ER stress and restore glucose homeostasis in a mouse model of type 2 diabetes. Science. 2006; 313(5790):1137-1140.

112.Nakamura $\mathrm{T}$, et al. Double-stranded RNAdependent protein kinase links pathogen sensing with stress and metabolic homeostasis. Cell. 2010;140(3):338-348.

113.Tabas I. The role of endoplasmic reticulum stress in the progression of atherosclerosis. Circ Res. 2010; 107(7):839-850.

114. Goldfine AB, et al. Use of Salsalate to Target Inflammation in the Treatment of Insulin Resistance and Type 2 Diabetes. Clin Transl Sci. 2008;1(1):36-43.

115. Hevener AL, et al. Macrophage PPAR gamma is required for normal skeletal muscle and hepatic insulin sensitivity and full antidiabetic effects of thiazolidinediones. J Clin Invest. 2007;117(6):1658-1669.

116. Larsen CM, et al. Interleukin-1-receptor antagonist in type 2 diabetes mellitus. New Engl J Med. 2007; 356(15):1517-1526.

117. Dominguez H, et al. Metabolic and vascular effects of tumor necrosis factor-alpha blockade with etanercept in obese patients with type 2 diabetes. $J$ Vasc Res. 2005;42(6):517-525.

118.Stanley TL, et al. TNF-alpha antagonism with etanercept decreases glucose and increases the proportion of high molecular weight adiponectin in obese subjects with features of the metabolic syndrome. J Clin Endocrinol Metab. 2011;96(1):E146-E150.

119. Ramsey SA, et al. Uncovering a macrophage transcriptional program by integrating evidence from motif scanning and expression dynamics. PLoS Comput Biol. 2008;4(3):e1000021. 